\title{
Incidence of Intracranial Melanoma Progression in the Setting of Positive Extracranial Response to Targeted Therapy and Immunotherapy: An Indication for More Frequent Screening in This Population?
}

Domenico A. Gattozzi ${ }^{1}$, Casey Rosso ${ }^{2}$, Bryan A. Schatmeyer ${ }^{1}$, Jean-Luc K. Kabangu ${ }^{1}$, Gary C. Doolittle ${ }^{3}$, Fen Wang ${ }^{4}$, Timothy Stepp ${ }^{1}$

1. Neurosurgery, University of Kansas Medical Center, Kansas City, USA 2. Neurosurgery, University of Kansas Medical School, Kansas City, USA 3. Hematology/Oncology, University of Kansas Medical Center, Kansas City, USA 4. Radiation Oncology, University of Kansas Medical Center, Kansas City, USA

Corresponding author: Domenico A. Gattozzi, dgattozzi@kumc.edu

\section{Abstract}

\section{Background and objective}

The incidence of intracranial metastases from melanoma is on the rise. In this study, we aimed to determine the incidence of intracranial disease progression in patients on BRAF/MEK targeted therapy and immunotherapy in the setting of controlled or improving extracranial disease.

\section{Methods}

This was a single-center, retrospective review that involved patients who underwent stereotactic radiosurgery (SRS) for intracranial metastatic melanoma between January 1, 2014, and December 31, 2018. We focused on BRAF/MEK mutation status and dates of treatment with BRAF/MEK targeted therapy, immunotherapy [ipilimumab (Yervoy), nivolumab (Opdivo), or pembrolizumab (Keytruda)], and combination targeted and immunotherapy.

\section{Results}

A total of 51 patients were enrolled: 36 males and 15 females. The average age of the patients was 58.6 years, and 26 among them were BRAF mutation-positive. Seventeen had prior surgery with SRS as adjuvant therapy. The other 34 had SRS as primary treatment. Forty-two patients had extracranial disease present at the time of SRS. There were 34 patients treated with targeted and immune therapy. Overall, 16 patients (47.1\%) demonstrated controlled or improving extracranial disease, and 18 (52.9\%) demonstrated progressing extracranial disease at the time of SRS. In the subgroup analysis, patients treated with BRAF/MEK targeted therapy demonstrated a $75 \%$ rate of extracranial disease control. The extracranial disease was controlled in $43.75 \%$ of patients on immunotherapy with intracranial progression, while it was controlled in $30 \%$ of patients on both BRAF/MEK targeted therapy and immunotherapy with intracranial progression. Sixteen patients (47.1\%) developed intracranial metastasis in our study while having a stable systemic disease with BRAF/MEK targeted therapy, immunotherapy, or a combination of the two.

\section{Conclusion}

Based on our findings, a systemic response to targeted therapy and immunotherapy does not necessarily parallel intracranial protection.

Categories: Dermatology, Neurosurgery, Oncology

Keywords: melanoma, intracranial, metastatic melanoma, metastases, stereotactic radiosurgery, immunotherapy, pembrolizumab, nivolumab, ipilimumab

\section{Introduction}

The incidence of melanoma has been on the rise worldwide, and it is a common cause of intracranial metastases [1]. The prevalence of intracranial metastases in melanoma can range from 10 to $40 \%$ during a patient's lifetime [2-4]. The emergence of advanced BRAF/MEK targeted therapy and immunotherapy have resulted in significant improvement in survival rates among advanced-stage melanoma patients [5-7]. Stereotactic radiosurgery (SRS) is a common treatment for brain metastases, and many of the intracranial lesions treated by SRS at our facility are metastases lesions [8-11]. Recently, we have noticed a trend among patients with melanoma treated with SRS at our institution: many develop purely intracranial disease in the absence of extracranial disease or the presence of controlled extracranial disease. Proactively treating 


\section{Cureus}

intracranial metastases not only leads to intracranial disease control but also provides survival benefits for patients with controlled extracranial disease at the time of development of brain metastasis $[12,13]$. The additional benefit from proactive SRS or surgery is maintained even in patients being treated with immunotherapy and targeted therapy [14].

In this study, we reviewed all cases of patients treated with SRS for melanoma metastases in an effort to quantify the degree to which BRAF/MEK targeted therapy and immunotherapy show an extracranial response with the development of intracranial metastatic diseases.

\section{Materials And Methods}

This study was approved by our institutional review board. It was a single-center, retrospective analysis of patients who received SRS as primary treatment for brain metastasis from metastatic melanoma. Subjects were compiled from the SRS database at our institution. Charts were accessed from the institution's electronic medical record system. All data was collected and de-identified prior to being reviewed by the authors. We calculated the data based on the date of the primary diagnosis of melanoma. All patients had resection of primary melanoma, allowing for the testing of BRAF mutation status of primary melanoma. We also analyzed the use of BRAF/MEK inhibitor therapy, immunotherapy, duration of systemic therapy before SRS, prior brain surgery, age at the time of SRS, the location and timing of extracranial disease burden, and whether extracranial disease burden worsened or improved on immune therapy.

\section{Results}

A total of 51 patients underwent SRS for intracranial melanoma metastases during the study period. Of these patients, 36 were males, 15 were females, and they ranged in age from 29 to 88 years old with an average age of 58.6. Table 1 presents the patient demographics and tumor characteristics. 


\section{Cureus}

Characteristics of the study population $(n=51)$

Patient sex

Male

$36(70.6 \%)$

Female

$15(29.4 \%)$

BRAF status

Positive

Negative

$N / A^{*}$

Surgery prior to SRS

Yes

No

$34(66.7 \%)$

Systemic disease present at the time of SRS

Yes

No

Age at the time of SRS

Average: $\mathbf{5 8 . 6}$ years

Range: 29-88 years

Primary tumor site

Scalp/face

Neck

Chest

Back

Extremities

\section{TABLE 1: Patient demographics and tumor characteristics}

*Did not undergo testing

SRS: stereotactic radiosurgery

Among the patients, $51 \%$ were positive for BRAF, $41 \%$ were negative, and $8 \%$ did not undergo testing. At the time of SRS, $42(82.4 \%)$ patients had extracranial disease in addition to their primary disease site, while nine (17.6\%) did not. Of the patients with the extracranial disease, 34 received systemic therapy prior to receiving SRS. Eight of the 34 patients (23.5\%) received only targeted therapy (BRAF/MEK inhibitors), 16 (47.1\%) received only immunotherapy prior to SRS, and $10(29.4 \%)$ received both targeted therapy and

immunotherapy before SRS. A total of 34 patients were on immunotherapy or targeted therapy prior to receiving SRS. We then determined which patients had progression of the extracranial disease (Table 2). 


\section{Cureus}

\begin{tabular}{|c|c|c|}
\hline \multicolumn{3}{|l|}{ Extracranial disease progression } \\
\hline \multirow{2}{*}{ Progression of extracranial disease on BRAF/MEK targeted therapy $(n=8)$} & Yes & $2(25 \%)$ \\
\hline & No & $6(75 \%)$ \\
\hline \multirow{2}{*}{ Progression of extracranial disease on immunotherapy $(n=16)$} & Yes & $9(56.25 \%)$ \\
\hline & No & $7(43.75 \%)$ \\
\hline \multirow{2}{*}{ Progression of extracranial disease on BRAF/MEK targeted therapy and immunotherapy $(n=10)$} & Yes & $7(70 \%)$ \\
\hline & No & $3(30 \%)$ \\
\hline \multirow{2}{*}{ Overall progression of extracranial disease $(n=34)$} & Yes & $18(52.9 \%)$ \\
\hline & No & $16(47.1 \%)$ \\
\hline
\end{tabular}

\section{Discussion}

Melanoma is known to have a potential for intracranial disease progression in the setting of positive extracranial response. This was initially reported pertaining to biomodulator therapies in the 1980s [15]. Recently, with the advent of BRAF/MEK inhibitors and immunologic therapies, the unmasking of intracranial metastatic disease from melanoma in the setting of systemic disease control has also been reported [16-22]. The presence of intracranial metastatic disease in melanoma is of diagnostic and prognostic importance. In fact, the most recent guidelines for melanoma staging from the American Joint Committee on Cancer have created the new category of "M1d" to specify the presence of central nervous system (CNS) metastatic disease burden [23].

The use of targeted and immunotherapies alone has not been able to provide an adequate response to intracranial metastases from melanoma. A recent phase 2 trial conducted in 2016 for pembrolizumab for patients with untreated brain metastases demonstrated 4/18 (22\%) patients with melanoma to have some degree of response to treatment [24]. The CheckMate204 study (a phase 2 trial) demonstrated a 26\% complete response rate, as well as a 30\% partial response rate, among 96 patients in the post-treatment phase with both nivolumab and ipilimumab after a median follow-up of 14 months [25]. One study cites only an approximate $50 \%$ response rate to immunotherapy intracranially, and even so, the intracranial response is not as prolonged as the extracranial response [26]. There have been multiple trials evaluating the efficacy of nivolumab, ipilimumab, or pembrolizumab for intracranial disease, yet the CheckMate204 trial has demonstrated the best response rates for intracranial metastatic melanoma by far [27]. There is also literature supporting the idea that the addition of SRS to immunotherapy as an initial treatment for brain metastases from melanoma is effective [28].

However, these studies do not address patients diagnosed with melanoma initially without intracranial disease and who then develop new intracranial disease after being placed on targeted therapies or immunotherapies. The use of each individual immunotherapy for patients with primary melanoma disease with undiagnosed/undeveloped intracranial metastasis is largely dependent on the patient's oncological provider, and the consideration of adverse side effects of the drug in the setting of a patient's overall condition and comorbidities. Therefore, regardless of the efficacy of each individual therapy or combination of therapies in treating known metastases, this study serves an important role in quantifying the development of intracranial metastatic disease in patients who are already on the therapeutic regimens. Our rationale for attempting to quantify this association between extracranial response with intracranial disease progression is to raise the question as to whether or not aggressive monitoring for intracranial disease in patients on BRAF/MEK targeted and immunologic therapies is required.

Our results show that almost half (47.1\%) of the patients treated with any combination of BRAF/MEK targeted therapy and immunotherapy develop intracranial disease in the setting of stable or improving extracranial disease, with only $52.9 \%$ having progressing extracranial disease at the time of clinical presentation. There is a difference in terms of survival in patients who have intracranial metastatic melanoma and lower functional status due to symptomatic disease compared to those with incidentally found disease [29]. In addition, the Graded Prognostic Assessment score for intracranial melanoma is lower, indicating lower median survival, for patients who are more severely symptomatic as evidenced by a lower Karnofsky Performance Scale [30]. It is therefore critical to understand and define the incidence of development of intracranial disease in patients on immunotherapies, and to our knowledge, our paper is the first of its kind to quantify this parameter in a patient population. 
Due to our small patient population, we were unable to reveal whether a single therapy or combination of therapies is more effective at intracranial protection. Also, since our study was retrospective and not prospective, we could not control for the decision as to why each patient received the treatment regimens they did, and in what order or combination. Overall, our data reveal that the patients who received both BRAF/MEK targeted therapy and immunotherapy show the highest degree of extracranial disease progression, at $70 \%$. This could be due to selection bias as ours was a population that was already selected for a more aggressive clinical course, and hence the requirement for two regimens attempted in the treatment course. Regardless, our study suggests that there is a significant incidence of intracranial disease burden from melanoma even in the setting of controlled or improving extracranial disease in patients while on BRAF/MEK targeted therapy and immunotherapies.

\section{Conclusions}

Sixteen patients (47.1\%) developed intracranial metastasis in our study while having stable systemic disease. This was observed in patients receiving BRAF/MEK targeted therapy, immunotherapy, or a combination of the two. This finding has ramifications for intracranial screening for patients with melanoma. Close intracranial monitoring and imaging follow-up should be conducted in metastatic melanoma patients on immunotherapy or a BRAF/MEK combination, even if they are asymptomatic.

Our data suggest that systemic response to therapy does not indicate intracranial protection. We have quantified the magnitude of this relationship for the first time and hopefully laid the groundwork for further studies. Larger studies will help us to better analyze the actual differences in the degree of intracranial protection between each of the treatments or combinations of treatments with more statistical certainty.

\section{Additional Information \\ Disclosures}

Human subjects: Consent was obtained or waived by all participants in this study. The University of Kansas Medical Center Institutional Review Board issued approval 0003352. This study was approved by the University of Kansas Medical Center Institutional Review Board. Animal subjects: All authors have confirmed that this study did not involve animal subjects or tissue. Conflicts of interest: In compliance with the ICMJE uniform disclosure form, all authors declare the following: Payment/services info: All authors have declared that no financial support was received from any organization for the submitted work. Financial relationships: All authors have declared that they have no financial relationships at present or within the previous three years with any organizations that might have an interest in the submitted work. Other relationships: All authors have declared that there are no other relationships or activities that could appear to have influenced the submitted work.

\section{Acknowledgements}

The authors would like to thank Joanne Conner for assisting in obtaining patient charts for this manuscript.

\section{References}

1. Lens MB, Dawes M: Global perspectives of contemporary epidemiological trends of cutaneous malignant melanoma. Br J Dermatol. 2004, 150:179-185. 10.1111/j.1365-2133.2004.05708.x

2. Staudt M, Lasithiotakis K, Leiter $\mathrm{U}$, et al.: Determinants of survival in patients with brain metastases from cutaneous melanoma. Br J Cancer. 2010, 102:1213-1218. 10.1038/sj.bjc.6605622

3. Nayak L, Lee EQ, Wen PY: Epidemiology of brain metastases. Curr Oncol Rep. 2012, 14:48-54. 10.1007/s11912-011-0203-y

4. Chiarion-Sileni V, Guida M, Ridolfi L, et al.: Central nervous system failure in melanoma patients: results of a randomised, multicentre phase 3 study of temozolomide- and dacarbazine- based regimens. $\mathrm{Br} \mathrm{J}$ Cancer. 2011, 104:1816-1821. 10.1038/bjc.2011.178

5. Theurich S, Rothschild SI, Hoffmann M, et al.: Local tumor treatment in combination with systemic ipilimumab immunotherapy prolongs overall survival in patients with advanced malignant melanoma. Cancer Immunol Res. 2016, 4:744-754. 10.1158/2326-6066.CIR-15-0156

6. Flaherty KT, Robert C, Hersey P, et al.: Improved survival with MEK inhibition in BRAF-mutated melanoma . N Engl J Med. 2012, 367:107-114. 10.1056/NEJMoa1203421

7. Robert C, Karaszewska B, Schachter J, et al.: Improved overall survival in melanoma with combined dabrafenib and trametinib. N Engl J Med. 2015, 372:30-39. 10.1056/NEJMoa1412690

8. Moreau J, Khalil T, Dupic G, et al.: Second course of stereotactic radiosurgery for locally recurrent brain metastases: safety and efficacy. PLoS One. 2018, 13:e0195608. 10.1371/journal.pone.0195608

9. Dziggel L, Bajrovic A, Schild SE, Rades D: Stereotactic radiosurgery alone for one to two brain metastases from cancer of unknown primary. Anticancer Res. 2018, 38:565-567. 10.21873/anticanres.12260

10. Vetlova E, Golbin DA, Golanov AV, et al.: Preoperative stereotactic radiosurgery of brain metastases: preliminary results. Cureus. 2017, 9:e1987. 10.7759/cureus.1987

11. Dahshan BA, Mattes MD, Bhatia S, Palek MS, Cifarelli CP, Hack JD, Vargo JA: Efficacy of stereotactic radiosurgery in patients with multiple metastases: importance of volume rather than number of lesions. Cureus. 2017, 9:e1966. 10.7759/cureus.1966

12. Gaudy-Marqueste C, Dussouil AS, Carron R, et al.: Survival of melanoma patients treated with targeted therapy and immunotherapy after systematic upfront control of brain metastases by radiosurgery. Eur J 
Cancer. 2017, 84:44-54. 10.1016/j.ejca.2017.07.017

13. Xu Z, Lee CC, Ramesh A, et al.: BRAF V600E mutation and BRAF kinase inhibitors in conjunction with stereotactic radiosurgery for intracranial melanoma metastases. J Neurosurg. 2017, 126:726-734. 10.3171/2016.2.JNS1633

14. Iorgulescu JB, Harary M, Zogg CK, et al.: Improved risk-adjusted survival for melanoma brain metastases in the era of checkpoint blockade immunotherapies: results from a national cohort. Cancer Immunol Res. 2018, 6:1039-1045. 10.1158/2326-6066.CIR-18-0067

15. Mitchell MS: Relapse in the central nervous system in melanoma patients successfully treated with biomodulators. J Clin Oncol. 1989, 7:1701-1709. 10.1200/JCO.1989.7.11.1701

16. McDonald MA, Sanghvi P, Bykowski J, Daniels GA: Unmasking of intracranial metastatic melanoma during ipilimumab/nivolumab therapy: case report and literature review. BMC Cancer. 2018, 18:549. 10.1186/s12885-018-4470-y

17. Mackiewicz J, Mackiewicz A: BRAF and MEK inhibitors in the era of immunotherapy in melanoma patients . Contemp Oncol (Pozn). 2018, 22:68-72. 10.5114/wo.2018.73890

18. Lipson EJ, Drake CG: Ipilimumab: an anti-CTLA-4 antibody for metastatic melanoma . Clin Cancer Res. 2011, 17:6958-6962. 10.1158/1078-0432.CCR-11-1595

19. Wolchok JD, Hodi FS, Weber JS, et al.: Development of ipilimumab: a novel immunotherapeutic approach for the treatment of advanced melanoma. Ann N Y Acad Sci. 2013, 1291:1-13. 10.1111/nyas.12180

20. Hazarika M, Chuk MK, Theoret MR, et al.: U.S. FDA approval summary: nivolumab for treatment of unresectable or metastatic melanoma following progression on ipilimumab. Clin Cancer Res. 2017, 23:34843488. 10.1158/1078-0432.CCR-16-0712

21. Barone A, Hazarika M, Theoret MR, et al.: FDA approval summary: pembrolizumab for the treatment of patients with unresectable or metastatic melanoma. Clin Cancer Res. 2017, 23:5661-5665. 10.1158/10780432.CCR-16-0664

22. Chuk MK, Chang JT, Theoret MR, et al.: FDA approval summary: accelerated approval of pembrolizumab for second-line treatment of metastatic melanoma. Clin Cancer Res. 2017, 23:5666-5670. 10.1158/10780432.CCR-16-0663

23. Gershenwald JE, Scolyer RA: Melanoma Staging: American Joint Committee on Cancer (AJCC) 8th edition and beyond. Ann Surg Oncol. 2018, 25:2105-2110. 10.1245/s10434-018-6513-7

24. Goldberg SB, Gettinger SN, Mahajan A, et al.: Pembrolizumab for patients with melanoma or non-small-cell lung cancer and untreated brain metastases: early analysis of a non-randomised, open-label, phase 2 trial. Lancet Oncol. 2016, 17:976-983. 10.1016/\$1470-2045(16)30053-5

25. Tawbi HA, Forsyth PA, Algazi A, et al.: Combined nivolumab and ipilimumab in melanoma metastatic to the brain. N Engl J Med. 2018, 379:722-730. 10.1056/NEJMoa1805453

26. Tétu P, Allayous C, Oriano B, et al.: Impact of radiotherapy administered simultaneously with systemic treatment in patients with melanoma brain metastases within MelBase, a French multicentric prospective cohort. Eur J Cancer. 2019, 112:38-46. 10.1016/j.ejca.2019.02.009

27. Kamath SD, Kumthekar PU: Immune checkpoint inhibitors for the treatment of central nervous system (CNS) metastatic disease. Front Oncol. 2018, 8:414. 10.3389/fonc.2018.00414

28. Minniti G, Anzellini D, Reverberi C, et al.: Stereotactic radiosurgery combined with nivolumab or Ipilimumab for patients with melanoma brain metastases: evaluation of brain control and toxicity. J Immunother Cancer. 2019, 7:102. 10.1186/s40425-019-0588-y

29. Kavouridis VK, Harary M, Hulsbergen AFC, et al.: Survival and prognostic factors in surgically treated brain metastases. J Neurooncol. 2019, 143:359-367. 10.1007/s11060-019-03171-6

30. Sperduto PW, Jiang W, Brown PD, et al.: Estimating survival in melanoma patients with brain metastases: an update of the Graded Prognostic Assessment For Melanoma Using Molecular Markers (Melanoma-molGPA). Int J Radiat Oncol Biol Phys. 2017, 99:812-816. 10.1016/j.ijrobp.2017.06.2454 